Original Article

\title{
Biomechanical analysis of INFINITY rehabilitation method for treatment of low back pain
}

\author{
Matej Daniel, PhD ${ }^{1}{ }^{*}$, Michaela Tomanová, PhD, MD²), Jana Hornová, MSc ${ }^{1)}$, \\ Iva NovotnÁ, MSc ${ }^{3)}$, Lenka Lhotské, PhD, MIEEE, MIET, CEng ${ }^{3)}$ \\ 1) Department of Mechanics, Biomechanics and Mechatronics, Faculty of Mechanical Engineering, \\ Czech Technical University in Prague: Technicka 4, 16000, Prague 6, Czech Republic \\ 2) Rehabilitation Clinic Bradýs nad Orlici, Czech Republic \\ 3) Czech Institute of Informatics, Robotics and Cybernetics, Czech Technical University in Prague, \\ Czech Republic
}

\begin{abstract}
Purpose] Low back pain is a pervasive problem in modern societies. Physical rehabilitation in treatment of low back pain should reduce pain, muscle tension and restore spine stability and balance. The INFINITY ${ }^{\circledR}$ rehabilitation method that is based on a figure of eight movement pattern was proved to be effective in low back pain treatment. The aim of the paper is to estimate the effect of a figure of eight motion on the L5/S1 load and lumbar spine muscle activation in comparison to other motion patterns. [Subjects and Methods] Three-dimensional model of lumbar spine musculoskeletal system is used to simulate effect of various load motion pattern induced by displacement of the center of gravity of the upper body. Four motion patterns were examined: lateral and oblique pendulum-like motion, elliptical motion and figure of eight motion. [Results] The simple pendulum-like and elliptical-like patterns induce harmonic muscle activation and harmonic spinal load. The figure of eight motion pattern creates high-frequency spinal loading that activates remodeling of bones and tendons. The figure of eight pattern also requires muscle activity that differs from harmonic frequency and is more demanding on muscle control and could also improve muscle coordination. [Conclusion] The results of the study indicate that complex motion pattern during INFINITY ${ }^{\circledR}$ rehabilitation might enhance the spine stability by influencing its passive, active and neural components.

Key words: Low back pain, Rehabilitation, Musculoskeletal model
\end{abstract}

(This article was submitted Oct. 19, 2016, and was accepted Feb. 7, 2017)

\section{INTRODUCTION}

Statistical reports from many developed countries indicate that low back pain is very common with almost half the adult population reporting low back pain lasting for at least 24 hours at some time in the year. Moreover, low back pain is a very frequent cause of long term disability in middle age ( 35 to 55 years of age $)^{1)}$. It is proposed that the most efficient prevention against low back pain is keeping movement activity supported with a suitable rehabilitation regime that does not overload the

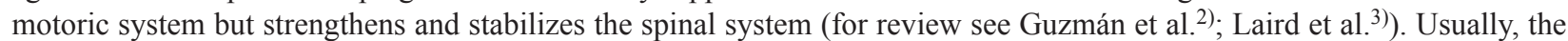
physical therapy involves supervised exercise therapy, spinal manipulative therapy or home exercise and advice ${ }^{4)}$.

A rehabilitation program should relieve pain and muscle tension; restore motion and balance; develop strength, endurance and power; as well as maintain cardiovascular fitness ${ }^{5}$. Traditional physical therapy or fitness training has emphasized strengthening of individual muscle groups. It has been proposed that the skill or the quality of the movement pattern that is

*Corresponding author. Matej Daniel (E-mail: matej.daniel@fs.cvut.cz; matej.daniel@gmail.com)

(C2017 The Society of Physical Therapy Science. Published by IPEC Inc.

This is an open-access article distributed under the terms of the Creative Commons Attribution Non-Commercial No Derivatives (by-nc-nd) License. (CC-BY-NC-ND 4.0: http://creativecommons.org/licenses/by-nc-nd/4.0/). 
used could be more important than achievement of muscle strength ${ }^{6}$. Stability and not strength can be considered as the objective in rehabilitation of low back pain ${ }^{7,8}$. Clinical studies also indicate improvements in disability degree and/or pain level using spine stabilization approach ${ }^{9}$. Various guidelines for treating low back pain have been proposed in different countries and regions ${ }^{10)}$. There is no therapy providing full recovery in all patients with low back pain yet and new therapeutic methods are being tested ${ }^{10,11)}$.

An original rehabilitation method named INFINITY ${ }^{\circledR}$ has been developed at the Rehabilitation Clinic Brandys nad Orlici, in the Czech Republic for low back pain therapy. The method has been verified in a clinical study ${ }^{12}$. Its name comes from the English word infinity because it utilizes movement in a figure of eight as a key segment of the exercises. The INFINITY ${ }^{\circledR}$ method is based on micro-movements in the range of millimeters and proved to be effective in patients with small spinal ROM (range of motion). The INFINITY ${ }^{\circledR}$ method can even be applied in the acute phase when a patient may be suffering from intense pain and it offers extended variability of autotherapeutic exercises. It was hypothesized that a figure of eight motion contributes to the stabilization and the strengthening of trunk muscles including the deep stabilization system. However, there seems to be a lack of science-based knowledge whether and how the figure of eight motion pattern during INFINITY ${ }^{\circledR}$ exercise influences spinal and muscular load.

Advances in mathematical modeling and technology are creating new opportunities that may quantify the effect of physiotherapy on lumbar spine ${ }^{13,14)}$. The overall aim of the study is to compare the effect of a figure of eight movement on deep muscle activation and spinal load with simple movements during rehabilitation. The spinal muscle and L5/S1 load is assessed using mathematical modeling.

\section{SUBJECTS AND METHODS}

Biomechanical modelling was adopted to estimate muscle and joint forces that are difficult to study through experimentation. The input into the biomechanical model is the anatomical model coupled with the physics of the musculoskeletal system. Considering the body static and dynamic equilibrium, active and passive internal forces (muscle and joint forces, respectively) acting within anatomical structure could be determined. Upright standing was taken as reference body posture within the present study and the spinal load is estimated considering equilibrium of gravitational, intra-abdominal, muscle and spinal reaction forces and torques. The L5/S1 junction was chosen as a point where spinal load is being estimated. The human body could be divided into two segments with respect to L5/S1: the part above L5/S1 junction, and the part below L5/ $\mathrm{S} 1$ junction, i.e. the upper body and the lower body, respectively (Fig. 1). Center of gravity of the upper body is located in front of the lumbar spine in upright standing and activity of postural muscles is needed to maintain equilibrium.

The musculoskeletal model of the lumbar spine described in Daniel ${ }^{13}$ ) is adopted (Fig. 1). Muscles attached over a large area were divided into individual musculoskeletal units according to Stokes and Gardner-Morse ${ }^{15)}$. Muscles with curved line of action were defined point-wise using wrapping points. The model contains 284 individual muscle units ${ }^{15}$.

The number of muscles included in the model exceeds number of equilibrium equations considerably. In principle, there are an infinite number of different combinations of muscle and joint forces that can be applied to maintain a particular posture

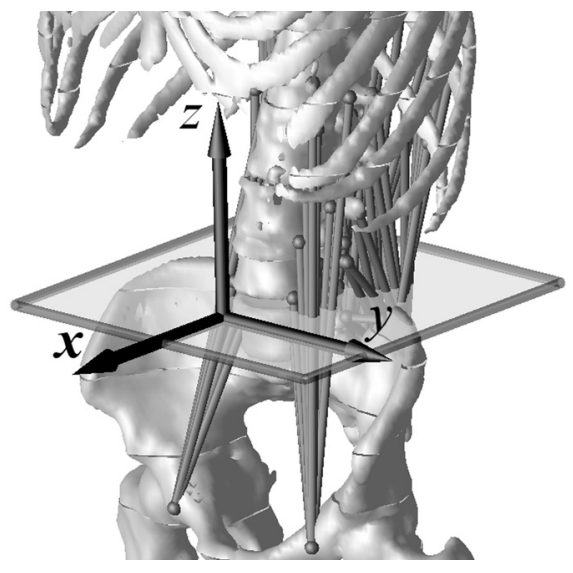

Fig. 1. Coordinate system and musculoskeletal model

Abdominal muscles are not shown. Origin of coordinate system corresponds to projection of center of gravity of upper body to L5/S1 transversal plane.
Table 1. Kinematic equations of the center of the upper body in the plane of section

\begin{tabular}{ll}
\hline \multicolumn{1}{c}{ Motion } & \multicolumn{1}{c}{ Kinematic equations } \\
\hline Linear L-R & $=0$ \\
& $=a_{y} \sin (\omega t)$ \\
& $x=a_{x} \sin (\omega t)$ \\
& $y=a_{y} \sin (\omega t)$ \\
Ellipsear AL-PR & $x=a_{x} \cos (\omega t)$ \\
& $y=a_{y} \sin (\omega t)$ \\
Lemniscate & $x=a_{x} \frac{2 \sqrt{2} \sin (\omega t) \cos (\omega t)}{1+\cos ^{2}(\omega t)}$ \\
$y$ & $=a_{y} \frac{\sin (\omega t)}{1+\cos ^{2}(\omega t)}$
\end{tabular}



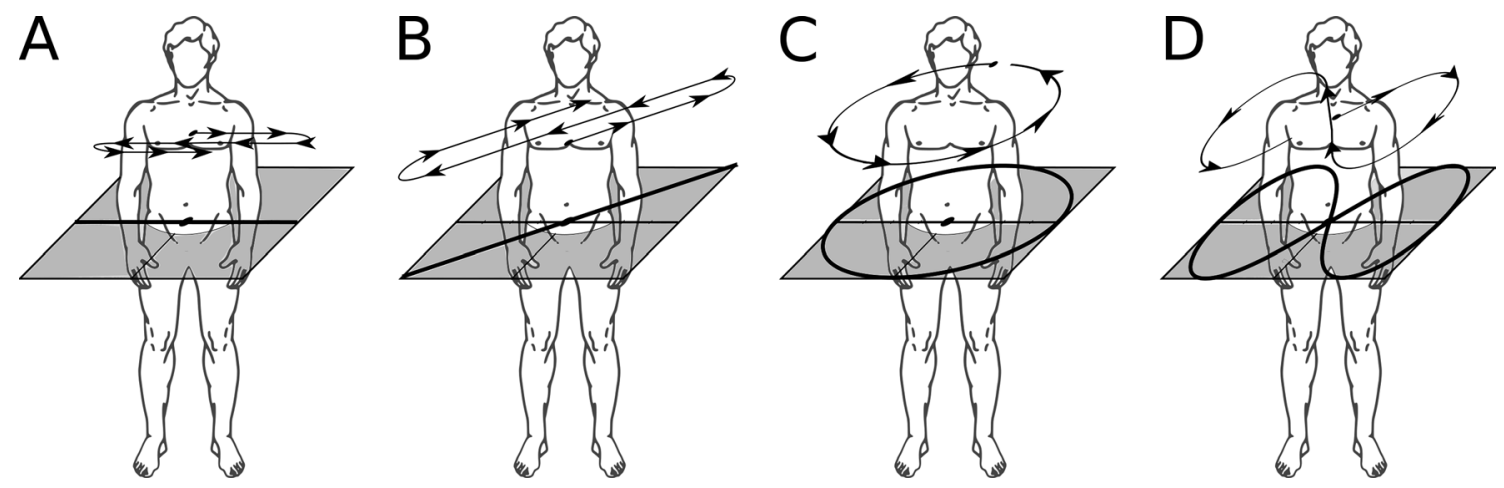

Fig. 2. Trajectories of upper body center of gravity and its projection into the transversal plane (A) L-R pendulum motion, (B) AL-PR pendulum motion, (C) ellipse motion, (D) $\infty$ shape motion. Displacements not in scale.

or produce a given movement ${ }^{16)}$. Therefore, the muscle activity required to maintain equilibrium in a given position of body is computed using the method of inverse dynamic optimization ${ }^{16}$. This method is based on assumption that loads are shared by muscles according to the minimization of some performance criterion. Minimization of the sum of muscle stresses cubed was used in this study as a performance criterion. This optimization criterion is based on the idea that muscles are activated in a way that minimizes the energy expenditure and maximizes their endurance time ${ }^{17}$ ). The force of each muscle is constrained not to exceed the maximum isometric force ${ }^{18)}$. After computation of the muscle forces, the components of the L4/S5 loading force are determined from the force equilibrium equations for the L5/S1 segment of spine. Magnitude of spine loading forces is used to express loading of the L5/S1 segment.

Rehabilitation motion pattern is represented by the motion of the center of gravity of the upper body with respect to its neutral position. Projection of the center of gravity in a neutral position of the body to a transversal plane passing the center of L5/S1 intervertebral disc is chosen as the center of the coordinate system (Fig. 1). Orientation of Cartesian coordinates is defined according to Stokes ${ }^{19)}$ : the $x$-axis points anteriorly, the $y$-axis points laterally and the $z$-axis points superiorly, defining four segments: anterior-left (AL), anterior-right (AR), posterior-left (PL), and posterior-right (PR) segment (Fig. 1).

Although the motion of the center of gravity of the upper body may follow a three-dimensional curve, the spine torque is influenced only by displacement of the center of gravity in the transversal (xy) plane. The periodical motion of the center of gravity of the upper body in the $x y$ plane during the exercise can be described by kinematic equation.

$x=a_{x} f(\omega t) ; y=a_{y} f(\omega t)$

where $\omega=2 \pi / T$ is the angular frequency with the period $T, f$ is function ranging from 0 to 1 and $a_{x}$ and $a_{y}$ are amplitudes of motion in $x$ and $y$ direction, respectively. As proposed by Schneck ${ }^{20)}$, the motion during the physical exercises can be described using simple harmonic functions (Table 1).

Four types of motion were modeled (Fig. 2, Table 1) and were chosen on the basis of our clinical experience. In upright standing a patient actively moves the center of gravity of their upper body along a specified curve. The linear L-R motion (Fig. 2A) and linear oblique motion AL-PR (Fig. 2B) represents simple pendulum movement in frontal and oblique planes, respectively. The circular motion is represented by ellipse trajectory (Fig. 2C) starting at anterior position. The most complex trajectory has figure of eight $(\infty)$ shape (Fig. 2D), denoted as lemniscate in algebraic geometry. A figure of eight is consistent with the INFINITY ${ }^{\circledR}$ method. A modified equation of Bernoulli lemniscate is used (Table 1).

There exist two principal types of INFINITY motion: the macro-movement (amplitudes in a range of centimeters) and the micro-movement (amplitudes in a range of millimeters) ${ }^{12}$. The period of movement depends on the movement range: the former has the period $T$ from 7 to $14 \mathrm{~s}$, while the periord $T$ is up to $5 \mathrm{~s}$ in the latter movement. Considering anteroposterior and mediolateral displacement $a_{x}$ and $a_{y}$ to be equal to $2 \mathrm{~cm}$ and $5 \mathrm{~mm}$ for macro and micro/movement respectively, the speed is lower than $0.05 \mathrm{~m} / \mathrm{s}$ and therefore inertial components are considered to be negligible, i.e. slow motion quasi-static analysis is performed ${ }^{13)}$. It is further assumed that small displacements of the center of gravity of the upper body do not significantly change musculoskeletal geometry of the lower lumbar spine, the motion occurs mainly in upper spine segments with larger mobility. Based on the INFINITY ${ }^{\circledR}$ method guidelines, the maximum anteroposterior (AP) displacement $\left(a_{x}\right)$ and left-right (LR) displacement $\left(a_{y}\right)$ was taken to be 2 and $4 \mathrm{~cm}$, respectively ${ }^{12}$. Calculations were performed for reference patient 65 kilograms weight and $170 \mathrm{~cm}$ height.

\section{RESULTS}

Figure 3 presents dependence of L5/S1 spinal load on general displacement of the center of gravity of the upper body. The 


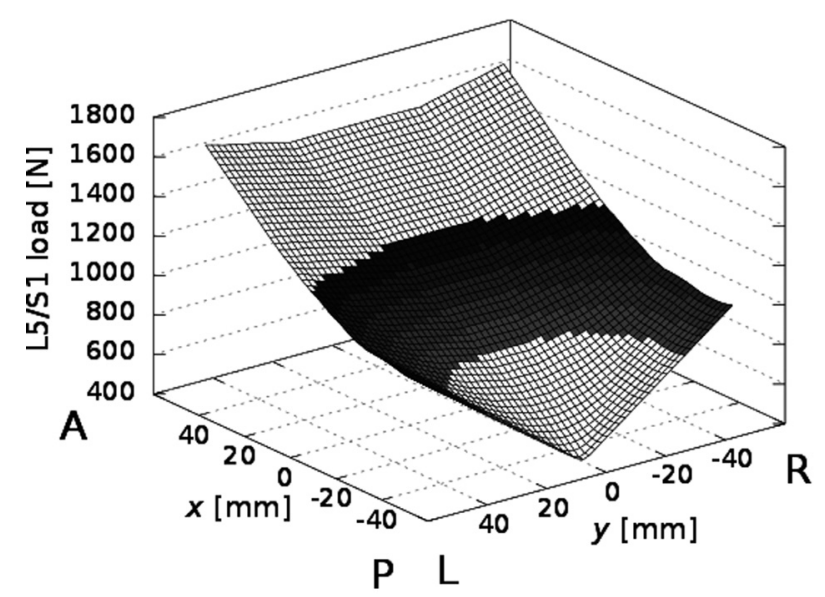

Fig. 3. Dependence between the L5/S1 spinal load and displacement of the upper body center of gravity in the transversal plane. Shaded area depicts zone of $10 \%$ deviation of spinal load from neutral position.

larger the anterior displacement $(x>0)$, the higher the spinal load. The larger the posterior displacement $(x<0)$, the lower the

spinal load. Spinal load is less sensitive to LR displacement $(y \neq 0)$ than to AP displacement. Within the studied range of motion, the spinal load is within 10\% deviation of load in neutral position (shaded area in Fig.3).

The values of spinal load for the four types of motion (Fig. 2, Table 1) are shown in Fig. 3 and Table 2. LR pendulum motion does not influence L5/S1 load (Fig. 4A). The range of spinal load is approximately the same in all studied motions except LR pendulum motion. The period of the spinal load cycle of AL-PR pendulum motion (Fig. 3B) and ellipse motion (Fig. 3C) is the same as the period of driving kinematics (Table 1) and the loading curve follows simple harmonic function. The $\infty$ shape displacements used in INFINITY ${ }^{\circledR}$ method exhibit two maxima and two minima of spinal force during one cycle (Fig. 3D), i.e. the frequency of spinal load cycle is twice the frequency of motion cycle.

The changes of muscle force in musculus iliocostalis is shown in Fig. 5. The magnitude of muscle force is almost the same in all studied motions. All studied motions except AL-PR pendulum motion load the left and right segments symmetrically, while AL-PR motion considerably overloads the right muscle segment. The frequency of muscle load follows the harmonic frequency of driving motion except the $\infty$ motion.

\section{DISCUSSION}

The effect of physiotherapy could be measured by means of spinal kinematics ${ }^{21)}$ or electromyography ${ }^{22)}$. However, measurement of muscle force of deep stabilizing muscles and spinal load is technically complex and invasive electromyography techniques are required ${ }^{23}$. The aim of this study was to estimate the effect of various motion patterns on load of musculoskeletal system of spine using a mathematical model. The benefit of biomechanical modelling is that it can test various motion pattern in controlled environment. The INFINITY method ${ }^{\circledR}$ has been proven as a suitable method in low back pain rehabilitation improving plantar functions, postural control in the upright stance and pain ${ }^{12}$.

Values of the spinal load proposed within this model are below the threshold proposed by NIOSH (1981) as low back injury risk in the workplace that is $3400 \mathrm{~N}^{24}$. Keeping the force low in rehabilitation of low back pain is required so as not to cause harm to already damaged tissues. In the patients with low back pain, the spinal force could be further elevated by increased muscle coactivation ${ }^{25)}$. We have found, that during micro-motions proposed by the INFINITY ${ }^{\circledR}$ method, the spinal load is not considerably increased. This provides rationale for application of the method in patients with a spine with low load bearing capacity.

A common belief is that muscle activation and muscle tension in physical therapy changes in direct response to specific interventions and enhances spinal stability ${ }^{26}$. This statement has been confirmed in the presented study: the more complex the motion pattern, the more complex the spine and the muscle loading cycle (Figs. 4, 5).

The study further indicates influence of complex motion on passive structures of muscles. $\infty$ motion pattern (Fig. 2D) activates passive structure by higher frequency loading (Fig. 4D) that is known to be associated with bone remodeling and maintaining bone health ${ }^{27)}$. Higher frequency load could also decrease the stiffness of viscoelastic tendons ${ }^{28)}$ and enhance spine mobility.

The symmetry of the loading cycle is important as well. Acute and chronic low back pain might be associated with 
Table 2. Maximum and minumum values of L5/S1 spinal load and musculus iliocostalis (ILC) force for various types of upper body center of gravity motion pattern

\begin{tabular}{lccccc}
\hline \multirow{2}{*}{ Motion } & $(\mathrm{A})$ & $(\mathrm{B})$ & $(\mathrm{C})$ & $(\mathrm{D})$ \\
\cline { 3 - 6 } & & Linear L-R & Linear AL-PR & Ellipse & $\infty$ shape \\
\hline \multirow{2}{*}{ Spine load } & Max [N] & 980.1 & $1,003.7$ & $1,003.6$ & $1,003.7$ \\
& Min [N] & 980 & 956.5 & 956.4 & 956.4 \\
\hline \multirow{2}{*}{ ILC load } & Max [N] & 172.2 & 178.8 & 173.9 & 172.2 \\
& Min [N] & 148.9 & 142.3 & 147.2 & 148.9 \\
\hline
\end{tabular}

A

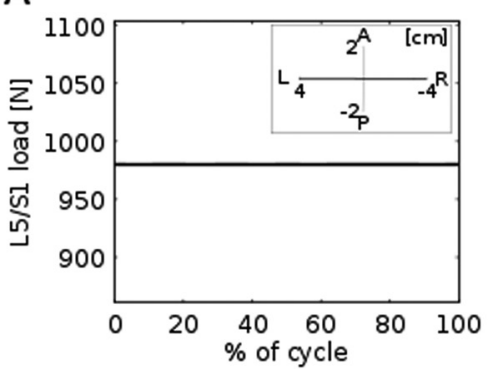

C

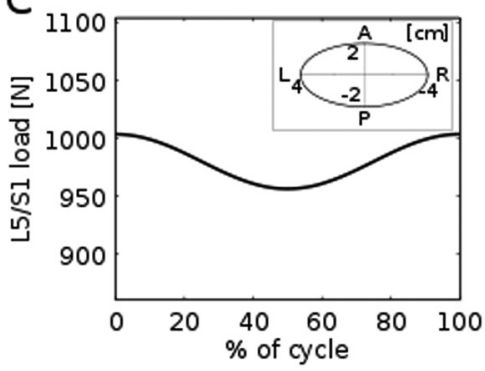

B

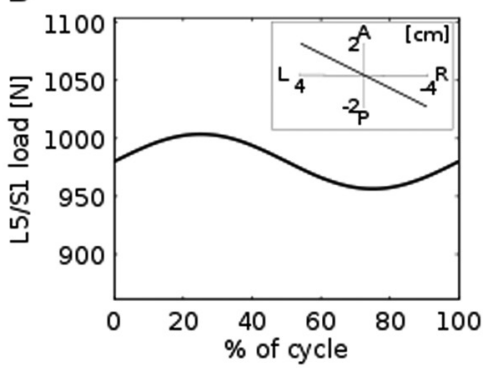

$D$

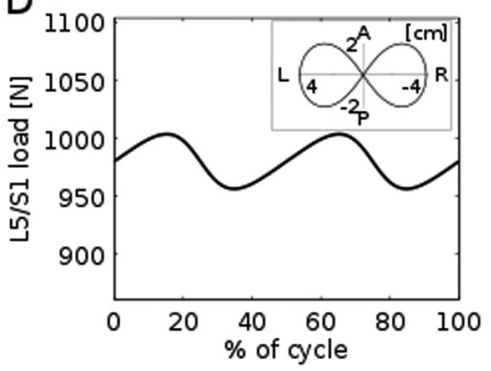

Fig. 4. L5/S1 spinal load for various types of upper body center of gravity motion pattern. Inset shows trajectories of center of gravity of upper body in the transversal plane for (A) L-R pendulum motion, (B) AL-PR pendulum motion, (C) ellipse motion, (D) $\infty$ shape motion.

ipsilateral atrophy of the multifidus muscles ${ }^{29)}$. Symmetric motion pattern conditions symmetric activation of the muscles (Fig. 5ACD) and hence strengthens the active structures symmetrically. Unsymmetrical motion pattern, e.g. oblique pendulum motion AL-PR, could overload right muscle segments (Fig. 5B). Asymmetric muscle loading predicted by the model in oblique AL-PR pendulum motion is in accordance with EMG measurements for asymmetric load ${ }^{30)}$. An interesting finding is that small LR pendulum motion could effectively exercise deep spine muscles without overloading the spine (Fig. 4A). This conclusion should be further verified in an EMG study.

Studied motion patterns were chosen on the basis of our clinical experience as they required active patient approach to muscle activation ${ }^{31)}$. Clinical study ${ }^{32)}$ indicates that active patient approach is significantly better than chiropractic spinal manipulation and home exercise in terms of patient satisfaction with treatment and trunk muscle endurance. Using an active patient approach offers extensive possibilities of autotherapy of motoric system and simultaneously brain training ${ }^{26)}$. While simple pendulum or ellipse motion pattern results in simple harmonic muscle activation pattern (Fig. 5), the more complex $\infty$ motion pattern requires a qualitatively more complex muscle activation. Complex muscle activation should be accompanied with active training of the central neural system, that could further increase spinal dynamic stability ${ }^{33)}$.

The mathematical model gives values of spinal load and pattern of EMG activation of back muscles that agree with the previous experimental measurements ${ }^{13)}$. However, a mathematical modeling approach presents several limitations. The model is limited to slow subtle motions that do not considerably change the lumbar musculoskeletal geometry. The spinal load could be increased considerably in case of large displacements due to lateral bending ${ }^{34)}$. The presented results are valid for subtle motions proposed in the INFINITY ${ }^{\circledR}$ method. Including the extensive motion in decimeters would require improvement of the muscle model, mainly by considering muscle wrapping of curved muscle ${ }^{35)}$. The mathematical model further assumes that muscles behave as the ideal force generators and underestimates the effect of nonlinear passive structures. Considering the muscle tendon dynamics and passive properties of the muscle-tendon unit would provide a more accurate force 
A

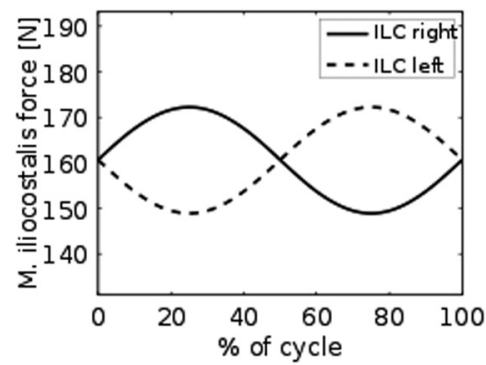

C

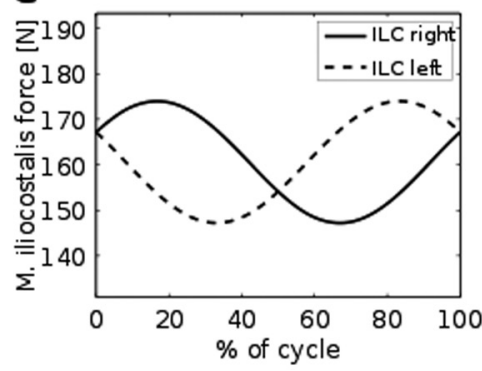

B

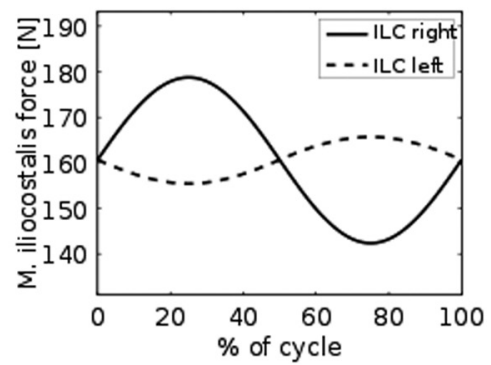

D

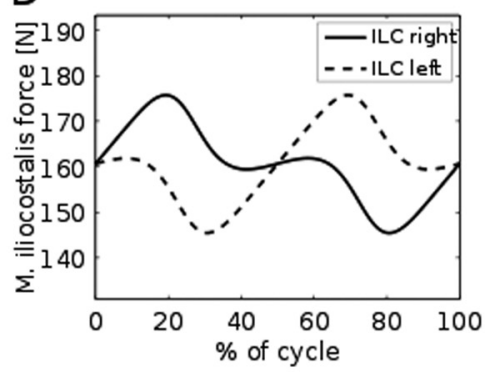

Fig. 5. Force in the left and right segments of musculus iliocostalis (ILC) for various types of upper body center of gravity motion pattern.

(A) L-R pendulum motion, (B) AL-PR pendulum motion, (C) ellipse motion, (D) $\infty$ shape motion.

estimation, especially for fast motions ${ }^{36)}$. To model the muscles force dynamics more accurately, a dynamics optimization approach which takes into account dynamic properties of neuromusculoskeletal systems should be used ${ }^{37)}$. The quasi-static model used in this study is in principle not able to fully consider the effects of motion, acceleration or stability goals on the muscle recruitment patterns of the trunk ${ }^{38)}$.

The model was designed to study the INFINITY ${ }^{\circledR}$ method that is primarily based on small micro-motions ${ }^{12)}$. Improvement in the mathematical model would allow its application in fast motion exercise, but would not influence the general conclusions of the study which shows the positive effect of a complex motion pattern. According to Panjabi theory ${ }^{39)}$, there are three components contributing to spinal stability: the passive system constituting bones, joints and ligaments; the active system constituting spine muscles and tendons; and the neural system comprising the nerves and central nervous system which direct and control the active system in providing dynamic stability. The study indicates that more complex motion provides higher frequency load to passive structure and requires complex activation of muscles enhancing neural system involvement. Results support hypothesis that the quality of the movement pattern that is used in rehabilitation is important to achieve spine stability ${ }^{6}$. General conclusions of the study are in agreement with clinical findings showing positive effect of complex Tai-Chi exercises on low back pain ${ }^{22)}$.

We conclude that the complexity of motion pattern directly influences the spinal load and muscle activation pattern. The simple pendulum-like or circular-like motions induce harmonic muscle activation and spinal load. The figure of eight pattern $(\infty)$ proposed in the INFINITY ${ }^{\circledR}$ method doubles the frequency of spinal loading while conditions complex muscle activation pattern that could induce remodeling in passive structures, strengthen active structures, and train the central neural system.

\section{ACKNOWLEDGEMENT}

The study was supported by the Medical Research Agency of the Czech Republic, project No. 15-33629A and Czech Technical University in Prague student grant No. SGS16/231/OHK3/3T/13.

\section{REFERENCES}

1) Burton AK, Balagué F, Cardon G, et al. COST B13 Working Group on Guidelines for Prevention in Low Back Pain: Chapter 2. European guidelines for prevention in low back pain: November 2004. Eur Spine J, 2006, 15: S136-S168. [Medline] [CrossRef]

2) Guzmán J, Esmail R, Karjalainen K, et al.: Multidisciplinary rehabilitation for chronic low back pain: systematic review. BMJ, 2001, 322: 1511-1516. [Medline] [CrossRef] 
3) Laird RA, Gilbert J, Kent P, et al.: Comparing lumbo-pelvic kinematics in people with and without back pain: a systematic review and meta-analysis. BMC Musculoskelet Disord, 2014, 15: 229. [Medline] [CrossRef]

4) Hayden JA, van Tulder MW, Tomlinson G: Systematic review: strategies for using exercise therapy to improve outcomes in chronic low back pain. Ann Intern Med, 2005, 142: 776-785. [Medline] [CrossRef]

5) Anderson MK, Parr GP, Hall SJ: Lumbar Spinal Conditions. In Found. Athl. Train, 4th ed. Lippincott Williams \& Wilkins, 2009, pp 306-347.

6) Janda V: On the concept of postural muscles and posture in man. Aust J Physiother, 1983, 29: 83-84. [Medline] [CrossRef]

7) Liebenson C: Musculoskeletal myths. J Bodyw Mov Ther, 2012, 16: 165-182. [Medline] [CrossRef]

8) Luque-Suárez A, Díaz-Mohedo E: Stabilization exercise for the management of low back pain. In Norasteh, AA (ed.), Low Back Pain, chapter 12. InTech 2010.

9) Hauggaard A, Persson AL: Specific spinal stabilisation exercises in patients with low back pain a systematic review. Phys Ther Rev, 2007, 12: 233-248. [CrossRef]

10) Kogure A, Kotani K, Katada S, et al.: A randomized, single-blind, placebo-controlled study on the efficacy of the arthrokinematic approach-hakata method in patients with chronic nonspecific low back pain. PLoS One, 2015, 10: e0144325. [Medline] [CrossRef]

11) Gordon R, Bloxham S: A systematic review of the effects of exercise and physical activity on non-specific chronic low back pain. Healthc (Amst), 2016, 4: 22. [Medline] [CrossRef]

12) Tomanova M, Lippert-Grüner M, Lhotska L: Specific rehabilitation exercise for the treatment of patients with chronic low back pain. J Phys Ther Sci, 2015, 27: 2413-2417. [Medline] [CrossRef]

13) Daniel M: Role of optimization criterion in static asymmetric analysis of lumbar spine load. Wien Med Wochenschr, 2011, 161: 477-485. [Medline] [CrossRef]

14) Dreischarf M, Zander T, Shirazi-Adl A, et al.: Comparison of eight published static finite element models of the intact lumbar spine: predictive power of models improves when combined together. J Biomech, 2014, 47: 1757-1766. [Medline] [CrossRef]

15) Stokes IA, Gardner-Morse M: Quantitative anatomy of the lumbar musculature. J Biomech, 1999, 32: 311-316. [Medline] [CrossRef]

16) Tsirakos D, Baltzopoulos V, Bartlett R: Inverse optimization: functional and physiological considerations related to the force-sharing problem. Crit Rev Biomed Eng, 1997, 25: 371-407. [Medline] [CrossRef]

17) Crowninshield RD, Brand RA: A physiologically based criterion of muscle force prediction in locomotion. J Biomech, 1981, 14: 793-801. [Medline] [CrossRef]

18) Stokes IA, Gardner-Morse M: Lumbar spinal muscle activation synergies predicted by multi-criteria cost function. J Biomech, 2001, 34: 733-740. [Medline] [CrossRef]

19) Stokes IA: Three-dimensional terminology of spinal deformity. A report presented to the Scoliosis Research Society by the Scoliosis Research Society Working Group on 3-D terminology of spinal deformity. Spine, 1994, 19: 236-248. [Medline] [CrossRef]

20) Schneck DJ: Engineering principles of physiologic function. New York: New York University Press, 1990.

21) Mieritz RM, Hartvigsen J, Boyle E, et al.: Lumbar motion changes in chronic low back pain patients: a secondary analysis of data from a randomized clinical trial. Spine J, 2014, 14: 2618-2627. [Medline] [CrossRef]

22) Cho Y: Effects of tai chi on pain and muscle activity in young males with acute low back pain. J Phys Ther Sci, 2014, 26: 679-681. [Medline] [CrossRef]

23) Albert WJ, Sleivert GG, Neary JP, et al.: Monitoring individual erector spinae fatigue responses using electromyography and near infrared spectroscopy. Can J Appl Physiol, 2004, 29: 363-378. [Medline] [CrossRef]

24) Potvin JR: Occupational spine biomechanics: a journey to the spinal frontier. J Electromyogr Kinesiol, 2008, 18: 891-899. [Medline] [CrossRef]

25) Marras WS, Davis KG, Ferguson SA, et al.: Spine loading characteristics of patients with low back pain compared with asymptomatic individuals. Spine, 2001, 26: 2566-2574. [Medline] [CrossRef]

26) Snodgrass SJ, Heneghan NR, Tsao H, et al.: Recognising neuroplasticity in musculoskeletal rehabilitation: a basis for greater collaboration between musculoskeletal and neurological physiotherapists. Man Ther, 2014, 19: 614-617. [Medline] [CrossRef]

27) Klika V, Marsik F: A thermodynamic model of bone remodelling: the influence of dynamic loading together with biochemical control. J Musculoskelet Neuronal Interact, 2010, 10: 220-230. [Medline]

28) Nordin M, Frankel VH, eds.: Basic biomechanics of the musculoskeletal system. Philadelphia: Lea \& Fibiger, 1989.

29) Wallwork TL, Stanton WR, Freke M, et al.: The effect of chronic low back pain on size and contraction of the lumbar multifidus muscle. Man Ther, 2009, 14: 496-500. [Medline] [CrossRef]

30) Oliveira AS, Gonçalves M: Lumbar muscles recruitment during resistance exercise for upper limbs. J Electromyogr Kinesiol, 2009, 19: 737-745. [Medline] [CrossRef]

31) Waddell G, Feder G, Lewis M: Systematic reviews of bed rest and advice to stay active for acute low back pain. Br J Gen Pract, 1997, 47: 647-652. [Medline]

32) Bronfort G, Maiers MJ, Evans RL, et al.: Supervised exercise, spinal manipulation, and home exercise for chronic low back pain: a randomized clinical trial. Spine J, 2011, 11: 585-598. [Medline] [CrossRef]

33) Szpala A, Rutkowska-Kucharska A, Drapala J: Electromechanical delay of abdominal muscles is modified by low back pain prevention exercise. Acta Bioeng Biomech, 2014, 16: 95-102. [Medline]

34) Marras WS, Granata KP: Spine loading during trunk lateral bending motions. J Biomech, 1997, 30: 697-703. [Medline] [CrossRef]

35) Hwang J, Knapik GG, Dufour JS, et al.: A biologically-assisted curved muscle model of the lumbar spine: model validation. Clin Biomech (Bristol, Avon), 2016, 37: 153-159. [Medline] [CrossRef]

36) Vilimek M: An artificial neural network approach and sensitivity analysis in predicting skeletal muscle forces. Acta Bioeng Biomech, 2014, 16: 119-127. [Medline]

37) Cadova M, Vilimek M, Daniel M: A comparative study of muscle force estimates using Huxley's and Hill's muscle model. Comput Methods Biomech Biomed Engin, 2014, 17: 311-317. [Medline] [CrossRef]

38) Knapik GG, Marras WS: Spine loading at different lumbar levels during pushing and pulling. Ergonomics, 2009, 52: 60-70. [Medline] [CrossRef]

39) Panjabi MM: The stabilizing system of the spine. Part II. Neutral zone and instability hypothesis. J Spinal Disord, 1992, 5: 390-396, discussion 397. [Medline] [CrossRef] 